\title{
ANÁLISE DOS FATORES ASSOCIADOS À SOBRECARGA DE CUIDADORES DE PACIENTES PORTADORES DA DOENÇA DE ALZHEIMER
}

\author{
ANALYSIS OF FACTORS ASSOCIATED TO OVERLOAD OF CAREGIVERS OF PATIENTS WITH \\ ALZHEIMER DISEASE
}

\section{Cristiana dos Santos ${ }^{{ }^{*}}$, Christiane Gabriel Bastos ${ }^{b^{*}}$, Francisco Ariclene Oliveira ${ }^{\mathrm{c}^{* *}}$, Denizielle de Jesus Moreira Moura ${ }^{\mathrm{d}^{*}}$}

acristianafametro@hotmail.com, bchrstianegaba@hotmail.com, cfranciscoariclene@hotmail.com, ddeniziellemoreira@gmail.com *Faculdade Metropolitana da Grande Fortaleza - Fortaleza (CE), Brasil **Hospital Geral Dr. César Cals - Fortaleza (CE), Brasil

\section{RESUMO}

Introdução: A sobrecarga do cuidador pode culminar no desenvolvimento de doenças agudas e crônicas e, consequentemente, no uso de diversas medicaçóes, tornando-o tão doente quanto o idoso com Alzheimer. Portanto, a avaliação do nível de sobrecarga do cuidador aponta uma direção mais efetiva para o profissional quanto às orientaçóes de cuidado. Objetivo: Analisar os fatores associados aos níveis de sobrecarga de cuidadores de portadores da doença de Alzheimer, fundamentados na Escala de Zarit. Materiais e método: Trata-se de um estudo transversal, realizado com 70 cuidadores. Os dados foram coletados no ambulatório de demência de um hospital terciário, por meio de formulário. Utilizou-se o Statistical Package for the Social Sciences (SPSS) para a análise estatística. Para avaliar a associação entre a variável dependente (nível de sobrecarga) e as independentes (características socioeconômicas e presença de doenças e/ou sintomas após ser cuidador), os testes utilizados foram razão de verossimilhança e qui-quadrado. Para comparação entre as médias utilizouse t-Student. Resultados: Verificou-se associação entre os níveis de sobrecarga e as variáveis grau de parentesco $(p=0,049)$, escolaridade $(p=0,029)$, renda familiar e renda per capita $(p=0,007)$, se adquiriu doença $(p=0,004)$ e sintomas físicos após ser cuidador $(p=0,030)$. As médias de participação financeira $(p=0,018)$, renda familiar $(p<0,001)$ e renda per capita $(p<001)$ foram maiores naqueles que tiveram menor intensidade de sobrecarga. Conclusóes: A identificação dos fatores que influenciam na sobrecarga do cuidador subsidia o planejamento de intervençóes para a prevençáo de doenças e a promoção da saúde, contribuindo para melhoria da sua qualidade de vida.

Palavras-chave: Doença de Alzheimer; demência; cuidadores; idoso.

\section{ABSTRACT}

Introduction: Caregiver's overload may culminate in the development of acute and chronic diseases and, consequently, in the use of various medications, making him/her as sick as the elderly with Alzheimer. Therefore, the assessment of the level of caregiver's overload points a more effective direction for the professional regarding the care guidelines. Objective: To analyze the factors associated with the overload levels of caregivers of patients with Alzheimer disease, based on the Zarit Scale. Materials and methods: This is a cross-sectional study carried out with 70 caregivers. Data were collected in the dementia outpatient clinic of a tertiary hospital, by form. The Statistical Package for Social Sciences (SPSS) was used for statistical analysis. To evaluate the association between the dependent variable (overload level) and the independent variables (socioeconomic characteristics and presence of diseases and/or symptoms after being a caregiver), the tests used were chi-square and likelihood ratio. Student's t-test was used to compare the means. Results: Association was verified 
between the levels of overload and the variables degree of kinship $(p=0.049)$, schooling $(p=0.029)$, family income and per capita income $(\mathrm{p}=0.007)$, if he/she has acquired disease $(\mathrm{p}=0.004)$ and physical symptoms after being caregiver $(\mathrm{p}=0.030)$. The averages of financial participation $(\mathrm{p}=0.018)$, family income $(\mathrm{p}<0.001)$ and per capita income $(\mathrm{p}<001)$ were higher in those with lower intensity of overload. Conclusions: The identification of factors that influence the caregiver overload subsidizes the planning of interventions for disease prevention and health promotion, while contributing to a better life quality.

Keywords: Alzheimer disease; dementia; caregivers; elderly.

\section{Introdução}

O tamanho da populaçáo idosa tem crescido em todo o mundo. Esse fenômeno demográfico tem gerado impacto nos setores da saúde, da economia/previdência, do transporte coletivo, dentre outros.

Os idosos estão sujeitos a uma série de mudanças próprias do envelhecimento, que lhes conferem olhar diferenciado, sobretudo no que se refere aos cuidados com a saúde. Surgem também alteraçóes físicas e psíquicas que ocasionam doenças, dentre as quais se pode citar a doença de Alzheimer como uma das afecçóes mais devastadoras para o idoso e seus familiares. $\mathrm{O}$ impacto do diagnóstico para a família pode ser desalentador, devido ao desconhecimento da doença, de o que fazer, de como agir, de como entender a pessoa afetada e, principalmente, de como entender os sentimentos em relação ao papel de cuidador ${ }^{1}$.

Atualmente, embora no Brasil existam lacunas estatísticas, a doença de Alzheimer é a principal causa de demência, cujo impacto é grande na sociedade, na vida do cuidador e do familiar. No país, a prevalência de demência tem atingindo $7,1 \%$ das pessoas acima de 65 anos de idade. Dentre estes pacientes, 54,1\% foram diagnosticados com a doença de Alzheimer ${ }^{2,3}$.

Nesse contexto, a família tem papel importante no cuidado com o doente, auxiliando-o no desempenho de atividades e no enfrentamento da doença. Faz-se necessário, portanto, orientar a família do cuidador acerca das mudanças que irão afetar seu cotidiano, além de propor estratégias para melhoria da qualidade de vida - sua e do doente.

O termo cuidador é atribuído à pessoa que auxilia quem necessita de cuidado físico no enfrentamento de doença e que não é capaz de se cuidar sozinho. Normalmente, a decisão de quem irá ocupar o papel de cuidador é feita informalmente e, muitas vezes, um familiar começa a assumir gradativamente as tarefas do paciente diante das necessidades que surgem ou, até mesmo, a partir do próprio paciente, que passa a delegar ou abdicar de suas atribuiçóes ${ }^{4}$.
Manter a rotina do idoso em casa ou no ambiente em que reside é de fundamental importância, uma vez que a rotina pode auxiliar na execuçáo das atividades da vida diária, reduzindo a sobrecarga do cuidador. Tomar conta de idosos pode ocasionar respostas negativas que influenciam no processo de cuidado e na relação cuidador-paciente ${ }^{3}$.

Nessa perspectiva, surgiram os questionamentos: qual o nível de sobrecarga do cuidador de paciente com Alzheimer? A sobrecarga gerada sobre os cuidadores ocasiona sintomas físicos ou psíquicos? Qual a relação entre o nível de sobrecarga e os fatores socioeconômicos?

A sobrecarga da doença nos cuidadores pode acarretar o desenvolvimento de sintomas psiquiátricos, fadiga, uso de medicamentos psicotrópicos, além da saúde prejudicada, o que ocasiona redução das condiçôes para cuidar do idoso 5 .

A sobrecarga do cuidador também pode culminar no desenvolvimento de doenças agudas e crônicas e, consequentemente, no uso de diversas medicaçôes, tornando-o táo doente quanto o idoso com Alzheimer. Portanto, a avaliação do nível de sobrecarga do cuidador traz uma direçáo mais efetiva para o profissional quanto às orientaçóes de cuidado e resulta em identificação precoce de possíveis doenças, bem como em intervençóes baseadas em conhecimentos científicos e comprometidas com o bem-estar do ser humano.

Assim, este estudo objetivou analisar os níveis de sobrecarga de cuidadores de portadores da doença de Alzheimer, fundamentando-se na Escala de Zarit, a qual permite avaliar a sobrecarga objetiva e subjetiva do cuidador informal e inclui informaçóes sobre saúde, vida social, pessoal, situação financeira, emocional e tipo de relacionamento ${ }^{6}$. Levou-se em conta que tal conhecimento pode contribuir para minimizar danos e desgastes de cuidadores, proporcionando-lhes melhor qualidade de vida por meio de cuidado individualizado e pautado em suas necessidades. 


\section{Métodos}

Trata-se de um estudo transversal, analítico, com abordagem quantitativa, realizado em um hospital terciário do Sistema Único de Saúde de Fortaleza, referência no ambulatório de demências.

A referida instituição conta com 91 cuidadores de pacientes com Alzheimer, os quais constituíram a população em estudo. Destes, três não consentiram participar da pesquisa, nove foram excluídos por serem cuidadores formais recebendo remuneração por esse serviço e nove eram residentes do interior do estado do Ceará, sendo excluídos do estudo. Dessa forma, a amostra foi constituída por 70 cuidadores informais, ou seja, pessoas (familiares ou náo) que desempenham a maior parte dos cuidados dispensados ao idoso e que não recebem remuneração por esse serviço.

Os dados foram coletados nos meses de outubro e novembro de 2014 na própria instituiçáo. Para execução da coleta, utilizou-se um formulário com dados socioeconômicos elaborado pelos autores e fundamentado nos Critérios de Classificação Econômica Brasil ${ }^{7}$. Nesse estudo, adotou-se a versão brasileira da Burden Interview-Zarit para classificação do nível de sobrecarga. Esse instrumento foi padronizado e validado por $S_{c a z u f c a}{ }^{6}$, baseado na versão original de Zarit, que tem por objetivo avaliar o impacto do ato de cuidar nas áreas: social, saúde, bem-estar emocional, situação financeira e relaçóes interpessoais. Quanto maior a pontuação recebida, maior a sobrecarga, classificada entre leve, moderada, moderada severa e severa.

Considerou-se o nível de sobrecarga como desfecho, sendo correlacionado com os preditores: sexo, idade, escolaridade, estado civil, renda, ocupação, grau de parentesco, doenças e/ou sintomas adquiridos após ser cuidador e o grau de dependência do idoso. Os testes para analisar essa associação foram os de Razáo de Verossimilhança, Qui-Quadrado $\left(\mathrm{x}^{2}\right)$ e Anova.

Posteriormente, os dados foram tabulados e analisados com o auxílio do Statistical Package for the Social Sciences (SPSS) 15.0. Após a consolidação dos resultados, a análise dos dados foi realizada à luz da literatura científica pertinente ao tema.

A pesquisa foi aprovada pelo Comitê de Ética em Pesquisa do Hospital Geral de Fortaleza com o $\mathrm{n}^{\circ}$ 442581, estando em conformidade com a Declaração de Helsinque ${ }^{8}$, revisada em 2000, e com a Resolução no 466, de 12 de setembro de 2012, do Conselho Nacional de Saúde?.

\section{Resultados}

Para melhor compreensão do nível de sobrecarga, fez-se necessária a caracterização dos aspectos socioeconômicos dos cuidadores, uma vez que estes influenciam sobremaneira no nível de sobrecarga. A caracterização da amostra evidencia que a maioria dos cuidadores é do sexo feminino (88,6\%); 65,7\% são filhos dos pacientes; $54,3 \%$ estão na faixa etária entre 22 e 49 anos, com uma média de 48,6 anos e desvio padrão de 11,52 , denotando pessoas em fase produtiva.

Quanto ao nível de escolaridade, observou-se que $2,9 \%$ são analfabetos, $35,7 \%$ têm o ensino fundamental incompleto/completo, $40 \%$ apresentam ensino médio incompleto/completo e $21,4 \%$ têm nível superior. Tal fato é condizente com a ocupação desempenhada pelos entrevistados, a saber, apenas $37,1 \%$ têm emprego formal. Todos relataram alguma atividade além da de serem cuidadores, quer sejam atividades do lar, estudantis ou empregatícias, acarretando para si uma sobrecarga ainda maior.

A renda familiar mostrou-se irregular, variando de $\mathrm{R} \$ 600,00$ a $\mathrm{R} \$ 6.000,00$. A renda per capita variou entre $\mathrm{R} \$ 120,00$ e $\mathrm{R} \$ 1.500,00$, com média de $\mathrm{R} \$ 567,80$. Observou-se a redução na renda quando se avaliou a renda per capita em virtude do número de dependentes do salário familiar. $\mathrm{O}$ número de dependentes variou de 2 a 8 , com uma média de $4,11 \pm 1,37$.

Todos os cuidadores apresentaram algum grau de sobrecarga, demonstrando as consequências da doença em sua qualidade de vida: $14,3 \%$ apresentaram sobrecarga moderada, $37,1 \%$ sobrecarga moderada severa e $48,6 \%$ sobrecarga severa. Tal fato pode estar relacionado à grande quantidade de pacientes em estágio avançado da doença, que exigem práticas de cuidado exaustivas.

Diante dessa realidade, é comum que o cuidador passe por cansaço físico, depressão, abandono do trabalho, alteraçôes na vida conjugal e familiar. A tensão e o cansaço sentidos pelo cuidador são prejudiciais náo só a ele, mas também à família e à própria pessoa cuidada.

O Gráfico 1 evidencia a presença de sinais e sintomas adquiridos após assumir a responsabilidade de cuidar de uma pessoa com Alzheimer. Observou-se a predominância dos sintomas psíquicos, demonstrando as repercussóes psicológicas advindas do ofício de cuidador. Dentre esses sintomas, destacam-se: estresse, nervosismo, ansiedade, depressão, preocupação, síndrome do pânico e esquecimento. 
Gráfico 1: Distribuição das queixas do cuidador após assumir o cuidado.

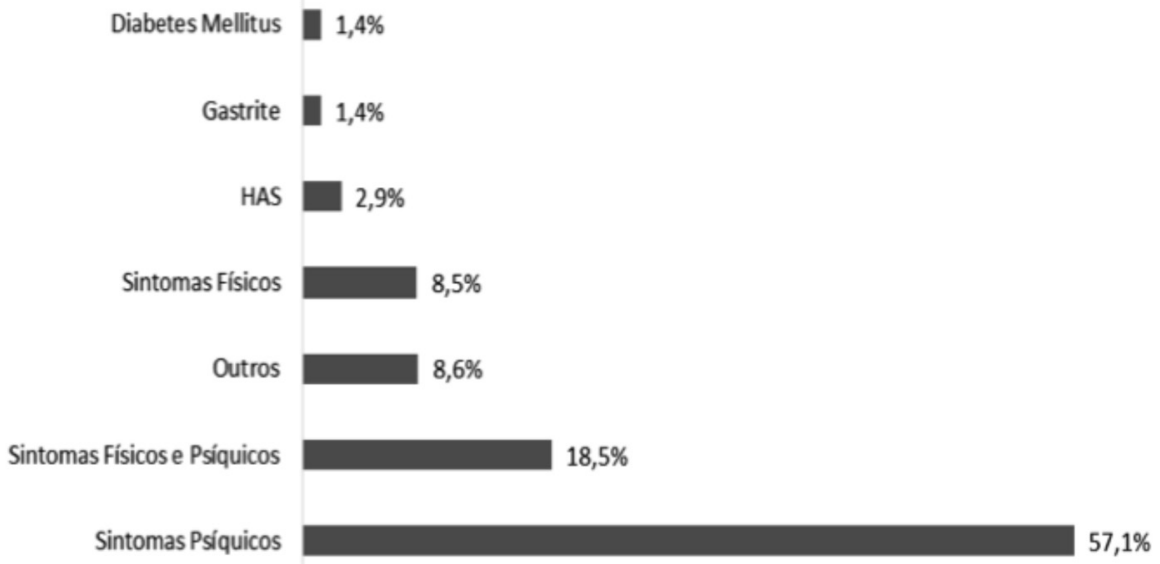

Fonte: Elaborado pelos autores, 2014.

A Tabela 1 apresenta a correlação entre as variáveis socioeconômicas e o nível de sobrecarga. Os testes para analisar essa associação foram o de Razão de Verossimilhança e Qui-Quadrado. Verificou-se associação $(\mathrm{p}<0,05)$ entre sobrecarga e as variáveis grau de parentesco $(\mathrm{p}=0,049)$, escolaridade $(p=0,029)$, renda familiar e per capita $(\mathrm{p}=0,007)$, presença de doença $(\mathrm{p}=0,004)$ e de sintomas físicos após ser cuidador $(\mathrm{p}=0,030)$.

Tabela 1: Descrição da correlação entre as variáveis socioeconômicas e o nível de sobrecarga do cuidador.

\begin{tabular}{|c|c|c|c|c|c|c|c|}
\hline \multirow{2}{*}{ Variáveis } & \multicolumn{2}{|c|}{ Sobrecarga moderada } & \multicolumn{2}{|c|}{ Sobrecarga moderada severa } & \multicolumn{2}{|c|}{ Sobrecarga severa } & \multirow{2}{*}{$\mathbf{p}$} \\
\hline & No & $\%$ & No & $\%$ & No & $\%$ & \\
\hline \multicolumn{8}{|l|}{ Sexo } \\
\hline Feminino & 10 & 16,1 & 23 & 37,1 & 29 & 46,8 & \multirow{2}{*}{0,251} \\
\hline Masculino & 0 & 0,0 & 3 & 37,5 & 5 & 62,5 & \\
\hline \multicolumn{8}{|l|}{ Faixa etária } \\
\hline $22-39$ & 2 & 14,3 & 9 & 64,3 & 3 & 21,4 & \multirow{5}{*}{0,077} \\
\hline $40-49$ & 3 & 12,5 & 5 & 20,8 & 16 & 66,7 & \\
\hline $50-59$ & 3 & 15,8 & 5 & 26,3 & 11 & 57,9 & \\
\hline $60-76$ & 2 & 15,4 & 7 & 53,8 & 4 & 30,8 & \\
\hline Média/SD & $49,20 \pm 12,5$ & & $47,77 \pm 12,5$ & & $49,06 \pm 10,6$ & & \\
\hline \multicolumn{8}{|l|}{ Estado Civil } \\
\hline Casado & 5 & 12,8 & 12 & 30,8 & 22 & 56,4 & \multirow{3}{*}{0,145} \\
\hline Solteiro & 4 & 16,7 & 13 & 54,2 & 7 & 29,2 & \\
\hline Divorciado/Viúvo & 1 & 14,3 & 1 & 14,3 & 5 & 71,4 & \\
\hline \multicolumn{8}{|l|}{ Ocupação } \\
\hline Do lar & 3 & 11,5 & 12 & 46,2 & 11 & 42,3 & \multirow{4}{*}{0,202} \\
\hline Estudante & 1 & 25,0 & 2 & 50,0 & 1 & 25,0 & \\
\hline Emprego formal & 6 & 23,1 & 7 & 26,9 & 13 & 50,0 & \\
\hline Autônomo & 0 & 0,0 & 5 & 35,7 & 9 & 64,3 & \\
\hline \multicolumn{8}{|c|}{ Grau de parentesco } \\
\hline Esposo(a) & 2 & 18,2 & 4 & 36,4 & 5 & 45,5 & \multirow{5}{*}{0,049} \\
\hline Filho(a) & 6 & 13,0 & 14 & 30,4 & 26 & 56,5 & \\
\hline Irmão(a) & 1 & 25,0 & 3 & 75,0 & 0 & 0,0 & \\
\hline Neto(a) & 0 & 0,0 & 5 & 83,3 & 1 & 16,7 & \\
\hline Outros & 1 & 33,3 & 0 & 0,0 & 2 & 66,7 & \\
\hline
\end{tabular}


Tabela 1: Continuação.

\begin{tabular}{|c|c|c|c|c|c|c|c|}
\hline \multirow{2}{*}{ Variáveis } & \multicolumn{2}{|c|}{ Sobrecarga moderada } & \multicolumn{2}{|c|}{ Sobrecarga moderada severa } & \multicolumn{2}{|c|}{ Sobrecarga severa } & \multirow[b]{2}{*}{$\mathbf{p}$} \\
\hline & No & $\%$ & No & $\%$ & No & $\%$ & \\
\hline \multicolumn{8}{|l|}{ Escolaridade } \\
\hline Analfabeto & 0 & 0,0 & 1 & 50,0 & 1 & 50,0 & \multirow{4}{*}{0,029} \\
\hline Fundamental & 2 & 8,0 & 11 & 44,0 & 12 & 48,0 & \\
\hline Médio & 2 & 7,1 & 13 & 46,4 & 13 & 46,4 & \\
\hline Superior & 6 & 40,1 & 1 & 6,7 & 8 & 53,3 & \\
\hline \multicolumn{8}{|c|}{ Renda em salário* } \\
\hline Até 2,5 & 1 & 4,0 & 10 & 40,0 & 14 & 56,0 & \multirow{3}{*}{0,007} \\
\hline $2,6-3,5$ & 1 & 4,2 & 11 & 45,8 & 12 & 50,0 & \\
\hline $3,6-8,8$ & 8 & 38,1 & 5 & 23,8 & 8 & 38,1 & \\
\hline \multicolumn{8}{|c|}{ No de dependentes } \\
\hline 2 a 3 & 4 & 16,0 & 11 & 44,0 & 10 & 40,0 & \multirow{3}{*}{0,813} \\
\hline 4 a 5 & 5 & 15,2 & 11 & 33,3 & 17 & 51,5 & \\
\hline 6 a 8 & 1 & 8,3 & 4 & 33,3 & 7 & 58,3 & \\
\hline
\end{tabular}

Fonte: Elaborado pelos autores, 2014.

*Valor do salário mínimo R $\$ 724,00$.

Apesar de os homens serem minoria neste estudo, observou-se neles maiores níveis de sobrecarga, haja vista que $62,5 \%$ apresentaram nível de sobrecarga severa. No entanto, não houve significância estatística na associação entre sexo e sobrecarga do cuidador $(\mathrm{p}=0,251)$.

A maioria dos cuidadores se encontra entre as faixas etárias de 40 a 49 e 50 a 59 anos. Identificou-se que os cuidadores dessas idades apresentam sobrecarga severa, com percentuais de $66,7 \%$ e $57,9 \%$, respectivamente. Considerando a associação idade e sobrecarga, obteve-se $p=0,077$. Ao analisar as médias de idade segundo o nível de intensidade da sobrecarga, detectou-se semelhança entre as médias, não havendo, portanto, significância estatística $(\mathrm{p}=0,900)$.

Os níveis de sobrecarga severa também foram superiores entre os autônomos $(64,3 \%)$, dentre os quais náo se observou sobrecarga moderada $(\mathrm{p}=0,202)$. Esses dados podem revelar a dificuldade em conciliar um emprego formal e as atividades de cuidador.

Não há consenso na literatura quanto à relação entre o grau de parentesco e a sobrecarga. Neste estudo identificou-se associação estatística, sendo os filhos e cônjuges os que apresentaram maior sobrecarga, com $56,5 \%$ e $45,5 \%$, respectivamente $(\mathrm{p}=0,049)$.

Quanto ao nível de escolaridade, os analfabetos apresentaram maior sobrecarga $(50 \%$ com sobrecarga moderada a severa e $50 \%$ com sobrecarga severa). Os maiores percentuais de sobrecarga severa foram entre os cuidadores de nível superior; entretanto, observaram-se nesse grupo valores diluídos entre as três classificaçóes $(40,1 \%$ apresentam sobrecarga moderada, $6,7 \%$ sobrecarga moderada a severa e $53,3 \%$ sobrecarga severa). Constatou-se significância estatística entre os níveis de sobrecarga e o grau de escolaridade $(\mathrm{p}=0,029)$.

Observou-se também que as médias de participação financeira, de renda familiar e de renda per capita foram maiores naqueles que tiveram menor intensidade de sobrecarga, com valores de $\mathrm{p}=0,018, \mathrm{p}<001 \mathrm{e}$ $\mathrm{p}<001$, respectivamente.

Quanto ao número de dependentes da renda familiar, encontram-se valores diluídos nos três níveis de sobrecarga, não havendo significância estatística $(\mathrm{p}=0,813)$. As médias de número de dependentes, segundo o nível de intensidade da sobrecarga, também foram semelhantes $(p=0,366)$.

A associação entre o surgimento de doenças ou sintomas após ser cuidador $(\mathrm{p}=0,004)$, a presença de sintomas físicos $(\mathrm{p}=0,030)$ e o nível de sobrecarga revela significância estatística, conforme Tabela 2. A maioria dos cuidadores que referiram sintomas psíquicos, físicos e gastrite apresentaram sobrecarga severa, com percentuais de $53,7 \%, 76,5 \%$ e $100,0 \%$, respectivamente. 
Tabela 2: Descrição da correlação entre as doenças e/ou sintomas adquiridos após ser cuidador, o grau de dependência do idoso e o nível de sobrecarga.

\begin{tabular}{|c|c|c|c|c|c|c|c|}
\hline \multirow{2}{*}{ Doenças/sintomas } & \multicolumn{2}{|c|}{ Sobrecarga moderada } & \multicolumn{2}{|c|}{ Sobrecarga moderada severa } & \multicolumn{2}{|c|}{ Sobrecarga severa } & \multirow{2}{*}{$\mathbf{p}$} \\
\hline & No & $\%$ & No & $\%$ & No & $\%$ & \\
\hline \multicolumn{8}{|l|}{ Adquiriu doença } \\
\hline Sim & 7 & 11,1 & 22 & 34,9 & 34 & 54,0 & 0,004 \\
\hline Não & 3 & 42,9 & 4 & 57,1 & 0 & 0,0 & \\
\hline \multicolumn{8}{|l|}{ Sintomas psíquicos } \\
\hline Sim & 7 & 13,0 & 18 & 33,3 & 29 & 53,7 & 0,287 \\
\hline Não & 3 & 18,8 & 8 & 50,0 & 5 & 31,3 & \\
\hline \multicolumn{8}{|l|}{ Dor } \\
\hline Sim & 0 & 0,0 & 1 & 100,0 & 0 & 0,0 & 0,367 \\
\hline Não & 10 & 14,5 & 25 & 36,2 & 34 & 49,3 & \\
\hline \multicolumn{8}{|l|}{ Hipertensão } \\
\hline Sim & 0 & 0,0 & 1 & 50,0 & 1 & 50,0 & 0,718 \\
\hline Não & 10 & 14,5 & 25 & 36,2 & 34 & 49,3 & \\
\hline \multicolumn{8}{|l|}{ Diabetes } \\
\hline Sim & 0 & 0,0 & 1 & 100,0 & 0 & 0,0 & 0,367 \\
\hline Não & 10 & 14,5 & 25 & 36,2 & 34 & 49,3 & \\
\hline \multicolumn{8}{|l|}{ Sintomas físicos } \\
\hline Sim & 1 & 5,9 & 3 & 17,6 & 13 & 76,5 & 0,030 \\
\hline Não & 9 & 17,0 & 23 & 43,4 & 21 & 39,6 & \\
\hline \multicolumn{8}{|l|}{ Gastrite } \\
\hline Sim & 0 & 0,0 & 0 & 0,0 & 1 & 100,0 & 0,482 \\
\hline Não & 10 & 14,5 & 26 & 37,7 & 33 & 47,8 & \\
\hline \multicolumn{8}{|c|}{ Pacientes dependentes } \\
\hline Total & 2 & 5,9 & 13 & 38,2 & 19 & 55,9 & 0,134 \\
\hline Parcial & 8 & 22,2 & 13 & 36,1 & 15 & 41,7 & \\
\hline
\end{tabular}

Fonte: Elaborado pelos autores, 2014.

\section{Discussão}

O predomínio do sexo feminino na amostra de cuidadores neste estudo remete a significados históricos e culturais do papel da mulher na sociedade. Esse dado indica uma realidade da cultura brasileira, na qual a mulher possuiria a aptidão de cuidar e fornecer o apoio necessário nas diversas situaçóes enfrentadas pela família ${ }^{3}$. As mulheres, sobretudo adultas jovens, têm se responsabilizado pela administraçáo domiciliar, familiar e ainda agregam o trabalho extradomiciliar ${ }^{10}$.

Na maioria das vezes, o cuidado fica a cargo da filha ou esposa ${ }^{3}$. O número menor de cônjuges em relação ao de filhos pode estar associado ao fato de que os cônjuges também sejam idosos, o que dificulta a possibilidade de prestarem cuidados.

O baixo nível de escolaridade pode contribuir para que o papel de cuidador seja atribuído às pessoas desse grupo, na medida em que se sabe que a inserção no mercado de trabalho formal é mais difícil para aqueles indivíduos com baixa escolaridade ${ }^{10}$. Assim, é mais provável que essas pessoas se dediquem aos serviços domésticos, e à tarefa de cuidar do familiar dependente como extensão dessa atividade.

Observou-se, neste estudo, associação entre a renda familiar/renda per capita e o nível de sobrecarga do cuidador. As doenças acarretam prejuízos financeiros pelos custos com remédios e tratamentos, que se somam aos gastos da rotina doméstica, ocasionando sobrecarga financeira. A despesa da doença de Alzheimer representa mais um fator estressante na gama de tarefas do cuidador, já que ele passa a administrar as questōes financeiras do idoso, além das suas. Por essa razão é que frequentemente os cuidadores contabilizam sua renda em conjunto com a dos idosos como medida para facilitar o manejo dos recursos, e com isso a renda familiar aumenta ${ }^{11}$.

Com relação à presença de doenças e sintomas nos cuidadores, ressalta-se que se trata de um estudo transversal, podendo essas classificaçóes se modificarem para melhor ou não, a depender dos cuidados dispensados a esse público. $\mathrm{O}$ cuidado deve estar voltado não apenas 
para o doente, mas também para o cuidador, o qual pode ser um paciente em potencial. Por isso, a necessidade de um cuidado de enfermagem singular pautado na integralidade e nas necessidades individuais.

Além do cuidado clínico-assistencial, o profissional de enfermagem deve desempenhar o cuidado educativo, oferecendo meios para que o grupo em estudo receba informaçóes sobre o Alzheimer, como ele afeta o paciente e quais são os cuidados necessários para promoçáo da saúde e da qualidade de vida. Essas estratégias, utilizadas de forma correta, poderão minimizar as consequências da sobrecarga cotidiana.

Os cuidadores, ao se perceberem sobrecarregados, tendem a sentir maiores níveis de tensâo e, consequentemente, a desempenhar funçôes aquém de sua capacidade, o que resulta em uma situação de cuidado desequilibrada, normalmente acompanhada por resultados insatisfatórios ${ }^{11}$.

A tarefa de cuidar do outro geralmente se soma às demais atividades do dia a dia. O cuidador fica sobrecarregado, pois muitas vezes assume sozinho toda a responsabilidade pelos cuidados. Soma-se a isso o peso emocional da doença, que incapacita e traz sofrimento a uma pessoa querida ${ }^{12}$.

Acrescente-se que os cuidadores de pacientes com doença de Alzheimer possuem maiores chances de ter sintomas psiquiátricos, maior frequência de conflitos familiares e problemas no trabalho, se comparados a pessoas da mesma idade que não exercem esse papel ${ }^{13}$.

Logo, o familiar cuidador, ao se dedicar integralmente ao idoso com doença de Alzheimer, pode se tornar estressado e cansado, física e mentalmente, o que tende a piorar se ele náo recebe ajuda de outras pessoas $^{14}$. Por isso, é imprescindível o olhar atento da equipe multiprofissional aos cuidadores, visto que cuidar de um familiar com demência é estressante, pode contribuir para doenças psiquiátricas e físicas e aumentar os riscos de morte ${ }^{11}$.

Os cuidadores podem estar mais sujeitos a contrair enfermidades devido a possíveis quedas de resistência imunológica do organismo relacionadas ao estresse e à ansiedade. Como consequência, pode-se observar também prejuízo nas relaçóes sociais, perda da autoestima, reações depressivas e muitos outros distúrbios, tanto físicos quanto psicológicos. As doenças diagnosticadas nos cuidadores que compuseram a amostra deste estudo (hipertensão arterial, diabetes mellitus e gastrite) podem estar relacionadas à presença do estresse, importante fator de risco para essas doenças.

Assim, destaca-se o cansaço como o principal sintoma físico identificado em cuidadores ${ }^{3}$. Em outro estudo, emergiram sintomas de depressão, ansiedade, dores musculares, cefaleias constantes, insônia, além de os cuidadores manifestarem que estavam acometidos de doenças crônicas, devido à ausência de apoio para enfrentar a sobrecarga física e mental do trabalho diário ${ }^{13}$.

Ademais, salienta-se que características como idade, escolaridade, grau de parentesco e renda estáo associadas com o nível de sobrecarga. Com relação à faixa etária, as mais afetadas com sobrecarga severa foram as de 40-49 e 50-59. Estas são consideradas faixas etárias produtivas, de pessoas que são responsáveis pela administração domiciliar, familiar e que ainda agregam o trabalho extradomiciliar ${ }^{11}$.

Desse modo, ressalta-se que é importante conhecer o grau de escolaridade, tanto do cuidador quanto do idoso, uma vez que o conhecimento sobre a doença pode facilitar o processo de cuidar ${ }^{15}$.

Em um estudo sobre cuidadores de pacientes com doença de Alzheimer, identificou-se que a imposição do papel de cuidador, a falta de apoio dos outros familiares, o grau de dependência do enfermo relacionada à patologia e o desgaste físico e psicológico são os principais fatores geradores de sobrecarga dos cuidadores ${ }^{16}$.

Em relação ao surgimento de doenças ou sintomas após assumir o cuidado, a presença de sintomas psíquicos foi significante nos que apresentaram nível de sobrecarga severa. Em estudo semelhante, os cuidadores que relataram possuir uma ou mais doenças obtiveram maiores níveis de sobrecarga ${ }^{11}$.

Quanto à avaliaçáo da qualidade de vida desses cuidadores, o estudo identificou insatisfação na capacidade de realizar atividades de lazer, o que possivelmente está relacionado ao cotidiano do familiar. $\mathrm{O}$ cuidador muitas vezes encontra-se limitado pela sobrecarga e pela falta de divisão da tarefa de cuidar ${ }^{17}$.

Neste cenário, torna-se fundamental que o enfermeiro desenvolva estratégias de educação em saúde com esses cuidadores, visando minimizar as consequências causadas por esse ofício. Para tal, faz-se necessário o entendimento dos aspectos biopsicossociais desses indivíduos, o que fornecerá subsídios para um cuidado integral e pautado nas necessidades individuais.

\section{Conclusão}

Todos os cuidadores apresentaram algum grau de sobrecarga, demonstrando as consequências da doença em sua qualidade de vida: $14,3 \%$ apresentaram sobrecarga moderada, $37,1 \%$ sobrecarga moderada severa e $48,6 \%$ sobrecarga severa.

Evidenciou-se associação estatística entre os níveis de sobrecarga e as variáveis grau de parentesco, escolaridade, renda familiar, renda per capita, se adquiriu doença e se adquiriu sintomas físicos após ser cuidador 
$(\mathrm{p}<0,05)$. Tais dados ressaltam que déficits de conhecimento e questóes financeiras influenciam sobremaneira na qualidade de vida do cuidador.

Os resultados deste estudo visam instigar os profissionais de saúde a intervirem precocemente, com vistas à prevençáo de doenças e à promoçáo da saúde nos cuidadores. Estes devem estar empoderados sobre o processo saúde-doença em questáo, contribuindo para o enfrentamento das dificuldades e dos desafios impostos. $\mathrm{O}$ alvo de cuidado da equipe multidisciplinar deve englobar o portador de Alzheimer, seu cuidador e os membros familiares mais próximos.

\section{Referências}

1. Santana RF, Almeida KS, Savoldi NAM. Indicativos de aplicabilidade das orientaçóes de enfermagem no cotidiano de cuidadores de portadores de Alzheimer. Rev Esc Enferm USP [Internet]. 2009;43(2):459-64.

2. Barbosa RL, Morais JM, Resck ZMR, Dázio EMR. O cuidador domiciliar de paciente idoso com mal de Alzheimer. Rev Rene. 2012;5(13):1191-6.

3. Camacho ACLF, Silva MDF, Espírito-Santo FH. Estratégias de suporte para prevenção de doença do cuidador familiar. Rev Enferm UFPE. 2012;6(9):2258-65.

4. Lenardt MH, Hautsch Willig M, Seima MD, Pereira LF. The health status and life satisfaction of caregivers of elderly with Alzheimer's. Colomb Med (Cali). 2011;42(2):17-25.

5. Gratão ACM, Vendrúscolo TRP, Talmelli LFS, Figueiredo LC, Santos JLF, Rodrigues RAP. Sobrecarga e desconforto emocional em cuidadores de idosos. Texto \& contexto Enferm. 2012;21(2):304-12.

6. Scazufca M. Brazilian version of the Burden Interview scale for the assessment of burden of care in carers of people with mental illnesses. Rev Bras Psiquiatr. 2002;24(1):12-7.

7. Associação Brasileira de Empresas e Pesquisa. Critério Brasil 2015 e atualização da distribuição de classes para 2016 [Internet]. 2015 [citado em 2015 set 17]. Disponível em: https://goo.gl/Ncbreu
8. Associação Médica Mundial. Declaração de Helsinque. Declaração para orientação de médicos quanto a pesquisa biomédica envolvendo seres humanos [Internet]. 1964 [citado em 2017 nov 1]. Disponível em: https:/goo.gl/ e79VQc

9. Brasil. Conselho Nacional de Saúde. Resolução no 466, de 12 de dezembro de 2012. Aprova normas regulamentadoras de pesquisas envolvendo seres humanos. Diário Oficial da Uniâo. Brasília, DF; 2013.

10. Amendola F, Oliveira MAC, Alvarenga MRM. Qualidade de vida dos cuidadores de pacientes dependentes no programa de saúde da família. Texto \& Contexto Enferm. 2008;17(2):266-72.

11. Seima MD, Lenardt MH. A sobrecarga do cuidador familiar de idoso com Alzheimer. Texto \& Contextos (Porto Alegre). 2011;10(2):388-98.

12. Barbosa BCAL, Koopmans FF, Silveira ACD. O desenvolvimento de competências do cuidador do paciente com Alzheimer a partir das ideias de Paulo Freire. Rev Augustus. 2010;14(29):35-44.

13. Pinto MF, Barbosa DA, Ferreti CEL, Souza LF, Fram DS, Belasco AGS. Qualidade de vida de cuidadores de idosos com a doença de Alzheimer. Acta Paul Enferm. 2009;22(5):652-7.

14. Inouye K, Pedrazzani ES, Pavarini SCI, Toyoda CY. Quality of life of elderly with alzheimer's disease: a comparative study between the patient's and the caregiver's report. Rev Latino-Am Enferm [Internet]. 2010;18(1):26-32.

15. Gaioli CCLO, Furegato ARF, Santos JLF. Perfil de cuidadores de idosos com doença de Alzheimer associado à resiliência. Texto \& Contexto Enferm [Internet]. 2012;21(1):150-7.

16. Baptista BO, Beuter M, Girardon-Perlini NMO, Brondani CM, Budo MLD, Santos NO. A sobrecarga do familiar cuidador no âmbito domiciliar: uma revisão integrativa da literatura. Rev Gaúcha Enferm [Internet]. 2012;33(1):147-56.

17. Borghi AC, Sassá AH, Matos PCB, Decesaro MN, Marcon SS. Qualidade de vida de idosos com doença de Alzheimer e de seus cuidadores. Rev Gaúcha Enferm [Internet]. 2011;32(4):751-8.

\section{Como citar este artigo:}

Santos C, Bastos CG, Oliveira FA, Moura DJM. Análise dos fatores associados à sobrecarga de cuidadores de pacientes portadores da doença de Alzheimer. Rev. Aten. Saúde. 2017;15(54):29-36. 